\title{
Li-Fi: An Expedition through Radiance- A Review
}

\author{
Radha Zode \\ M.Tech, Department of Electronics Engineering, \\ G. H. Raisoni College of Engineering, Nagpur, India
}

\author{
Trushna Deotale \\ Prof, Department of Electronics Engineering, \\ G. H. Raisoni College of Engineering, Nagpur, India
}

\begin{abstract}
Li-Fi stands for Light- Fidelity. Li-Fi is a wireless communication system which uses light as a carrier instead of radio frequencies which is used in Wi-Fi. Both the $\mathrm{Li}-\mathrm{Fi}$ and Wi-Fi works on electromagnetic spectrum for the transfer of the data. Many Investigators have been currently working on Li-Fi technology for its promising features like security and speed has made this expertise a flourishing over Wi-Fi. Lightemitting diodes are used for the visible light transmission. It offers many advantages like working across higher bandwidth as well as higher transmission speed. LED devices are used only for radiance purpose by applying constant current. In this paper, an attempt is made to transmit a real time information using $\mathrm{Li}-\mathrm{Fi}$ technology.
\end{abstract}

\section{Keywords}

Wi-Fi, Li-Fi, light emitting diode, solar cell

\section{INTRODUCTION}

In this epoch of wireless communication, $\mathrm{Li}$-fi is a new mode of communication which uses LED lights to transmit data wirelessly. Transmission of data is the most important day to day activity in today's world. In current wireless creation, when multiple devices are connected to the same internet, its speed decreases proportionally. The availability of fixed bandwidth makes it much more difficult for the high data transfer rate and to connect a secure network with the increase in a number of devices.

The term Li-Fi was devised by German physicist Harald Hass in his 2011 TED Global talk. Li-Fi is light based Wi-Fi which uses light to transmit information whereas in $\mathrm{Wi}-\mathrm{Fi}$ radio waves are prerequisite.

A LED device is a low cost, secure and safe way to transmit data, and there is plenty of free visible light spectrum. Highintensity LED's used in light bulbs, flashlights and cameras can transmit very high data rate faster than Wi-Fi.

The figure below shows the environment with the Li-Fi technology where light bulbs are used as a communication medium to mobiles, PC, laptops as it all have photo detector at the receiver end.

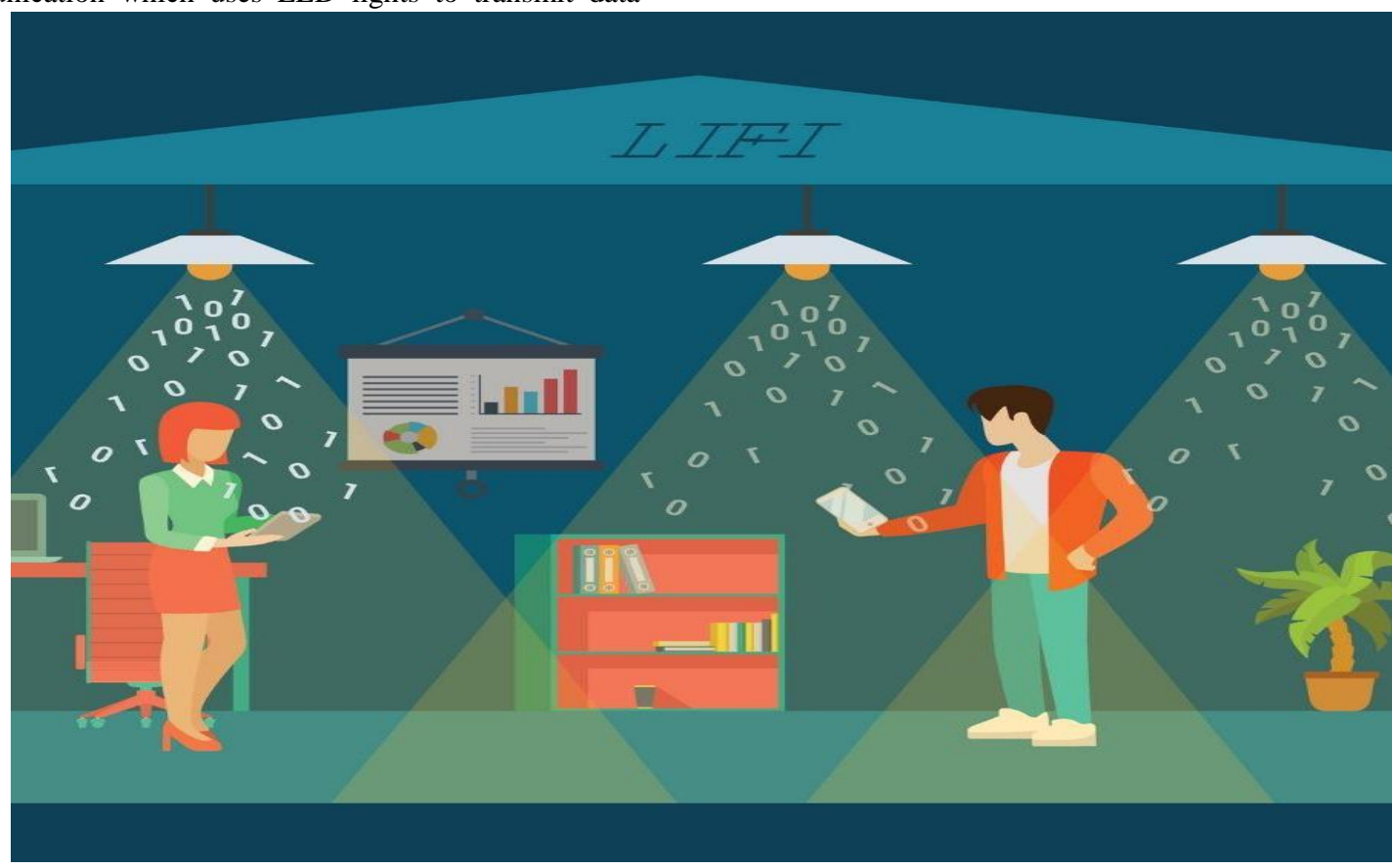

Fig 1: Layout of Li-Fi Technology

\section{LITERATURE REVIEW}

It is well-known fact that many people use Wi-Fi network to accomplish their task through a wired or wireless network. Though Wi-Fi gives us speed of $150 \mathrm{Mbps}$, it is still unsatisfactory to accommodate a number of desired users. WiFi provides us with internet access wirelessly. In the past few years, Wi-Fi technology is one of the most famous technologies used in school, colleges and even in public residences.
Dr. Harald Hass introduced the concept of Li-Fi. He explains how Li-fi technology is superior to Wi-Fi technology. His research directed many people to work on this forthcoming technology. He explains the $\mathrm{Li}-\mathrm{Fi}$ and argues with the 5G technology. It gives the information about transmission speed of using single micro LED. Ravi R. Nimbalkar et.al [3] explains how depending upon the data which want to encode, one can vary the LED flickering. It contains the information about diverse data channel with each frequency scrambling 
conditional upon the LED's that modifies the frequency. R. Mahendran [11] states about low cost, easy data transmission and delivers how the trustworthy communication is established. Mostafa Z. Afgani et.al [12] high PAR in OFDM and can be exploited constructively in visible light communication to modulate the intensity. The transmission results manage a distance close to one meter with the bit error of $10^{-3}$ under the adequate light circumstances. Mohit Vasuja et.al [13] explains the aspiration of the research paper is cunning a prototype of Li-FI transmitter and receiver to transmit data. After that Nidhi Soni et.al [2] explains how Li$\mathrm{Fi}$ is very much similar to $\mathrm{Wi}-\mathrm{Fi}$ and how the intensity of light emitting diode is used for transmission. It is evidenced that intensity is inversely relational to the square of the distance from the source. Speed rate of each type of LED's is different. Laser light offers maximum speed whereas RGB offers lowest rate. Normal LED provides the intermediate rate. Jay $\mathrm{H}$. Bhut et.al [6] solves the issue of shortage of radio frequency bandwidth by using the array of LED for parallel transmission. By this method, it is intended to create new communication channel using surviving equipment. Rahul R. Sharma et.al [4], emphases on developing a Li-Fi based system and evaluates the performance of the system with the existing technology. Ravi Prakash et.al [5] explains how LED illumination can be used as a communication source by modulating LED light with the data signal. This paper gives the information about high data rate by using high speed LED as well as by using a mixture of R, G, B LED to alter the light frequency with each frequency encrypting a diverse data channel. Such expansions promise to ideally escalation the speed. According to Akshata M Sonnad, Anjana Gopan, Sailakshmi N R, Divya S, Ambika R [14]. Transmission of data from one device to other using LEDs, thereby establishing a Li-Fi network in a localized environment. ONOFF modulation technique is used to transmit data through LED light. At the receiver end, photo detector demodulates the incoming received signal based on the sequence of 1 's and 0 's. This demodulated signal is then conducted to a filter to eradicate objectionable clatter. This cleaned signal is then improved using signal amplification mechanism. As per Shinichiro Haruyama in [7], Communication using visible light is an emerging province where visible light is used as a medium for the transmission of data. This paper focuses on the application like location-based services photodiode as well as an image sensor. They also mention properties of VLC as well as advantages and disadvantages. According to Ying jie He et.al [8], It emphases VLC archetype strategy with an enormous increase in transmission distance and upgrading in channel capacity. Transmission of quality video/audio images having a distance of $3 \mathrm{~m}$ can be reached and improvements can be made by adding a fixative lens between transmitter and receiver. According to $\mathrm{M}$. Mutthamma [9], $\mathrm{Li}-\mathrm{Fi}$ is a superset of altered wireless technologies such as cloud computing, communication, positioning. Analysis on speed and safekeeping of the li-fi system. As per Jin-Shyan Lee et al. [10], evaluation of different wireless communication standards and evaluate transmission time, coding efficiency, complexity, and power consumption.

\section{WI-FI}

As far as radiance is concern, $\mathrm{Li}-\mathrm{Fi}$ and $\mathrm{Wi}-\mathrm{Fi}$ are considered as best compatiting technologies. For that there is a comparison between both the technologies.

Wi-Fi stands for "wireless fidelity". Another name for Wi-Fi is WLAN(Wireless Local Area Network). Wi-Fi is a wireless interacting technology that routines radio waves as a carrier to transmit the data. It is most prominent technology in past few years. Wi-Fi is merely a trademarked expression that means IEEE 802.11x. Wi-Fi networks have wireless connection amongst sender and receiver by means of radio frequency. Wi-Fi affords wireless connectivity to the standing devices by releasing frequencies between $2.4 \mathrm{GHz}-5 \mathrm{GHz}$, dependent on the amount of data on the network. It is used for airing the internet with high speed when connected to an access point. This system of the signal range depends on the frequency band, radio power output, and modulation techniques. The standard antennas have reached up to $100 \mathrm{~m}$. Installation of $\mathrm{Wi}-\mathrm{Fi}$ is easy. A wireless router is required for setting up the Wi-Fi network. This router behaves as a hub for the wireless network. The router receives the signal and decode it and send the data to the internet by Ethernet connection. The router receives the data from the internet and translate it into the radio signal and will refer to the devices having wireless adaptors.

There are different Wi-Fi technologies having different bandwidth.

\section{Wi-Fi Generations}

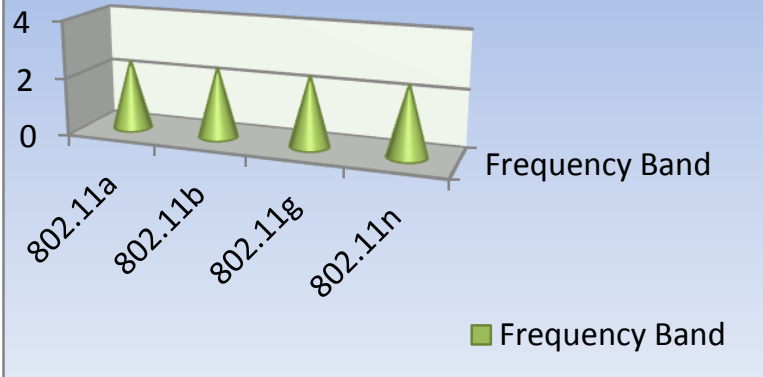

Fig 2: Wi-Fi generation with the frequency band

These generations comprehend diverse data speed which is logged in Mbps. The graph shows different data rate according to generations.

\section{Maximum Data Rate}

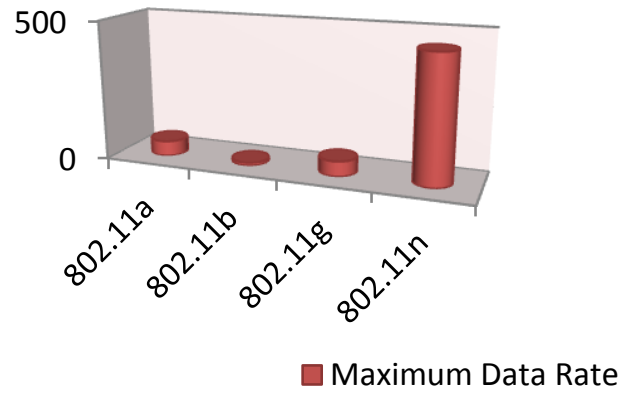

Fig 3: Maximum Data Rate

Wi-Fi has its identifiable disadvantages which lead to the innovation of $\mathrm{Li}-\mathrm{Fi}$ which are as follows: 


\subsection{Availability}

$\mathrm{Wi}-\mathrm{Fi}$ is not available in areas such as aircraft, medical applications, petrochemical plants because of the unavailability of radio signals.

\subsection{Capacity}

As wireless data is conducted through radio waves which are inadequate and expensive. Because of radio waves, this technology has restricted capacity.

\subsection{Efficiency}

Numerous radio base stations consume the maximum amount of energy as well as it necessitates more amount of energy for cooling down the base stations. Thus the efficiency of the base station is only $5 \%$.

\subsection{Security}

As radio waves can penetrate through walls. So, they can be intercepted and misused.

\section{DESIGN OF LI-FI}

The components required to design the system based on $\mathrm{Li}-\mathrm{Fi}$ are LED which serves as a communication source, Solar cell which serves as a receiving element, PDA, Laptops. While designing the Li-Fi system, some factors have to be taken into consideration are as follows:
1. Line of sight
2. High brightness LED
3. Presence of light

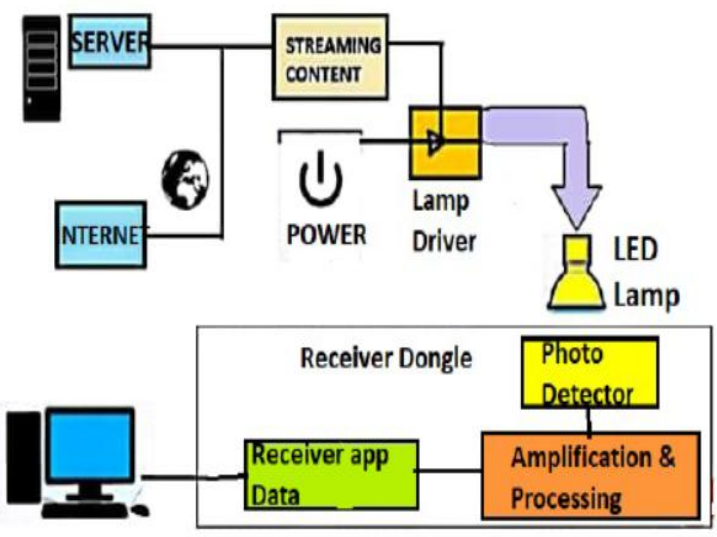

Fig 4: Architecture of Li-Fi

\section{FLOWCHART}

\section{STEP 1: - START}

STEP 2: - Define Device A and device B

STEP 3: - Make connections between device A and device B using hardware $\mathrm{H}$

STEP 4: - Input the data as "Li-Fi Technology" in device A

STEP 5: - Data is sent or transmitted to the hardware $\mathrm{H}$

STEP 6: - Hardware convert data into binary format

If LED is ON data 1 is transmitted to device B

If LED is OFF data 0 is transmitted to device B

STEP 7: - Data is transmitted to device B through hardware $\mathrm{H}$ with the help of light
STEP 8: - "Li-Fi Technology" is displayed as output on device $\mathrm{B}$

\section{STEP 9: - STOP}

\section{IMPLEMENTATION OF LI-FI}

The implementation of Li-Fi is done by using white LED at the downlink transmitter. LED devices are used only for illumination purpose by applying a constant current across it. By varying the input current, the output made vary at very high speed. It works as, when the LED is ON, logic 1 is transmitted and when the LED is OFF, logic 0 is transmitted. LED's flickering occurs at a very fast rate and which cannot follow by the human eye. In this method, parallel data transmission could be possible through the use of an array of an LED. The light signal is received by the solar cell at the receiver end. The converse method takes place at the destination computer to regain the data back from the received light. LEDs are employed as the light sources. Direct modulation of the light is used to spread digital signal. The emitted light is detected at the receiver.

Source Computer: Data Reading Module $\rightarrow$ Data Conversion Module $\rightarrow$ Transmitter Module

Destination Computer: Receiver Module $\rightarrow$ Data Interpretation Module $\rightarrow$ Data Display (GUI)

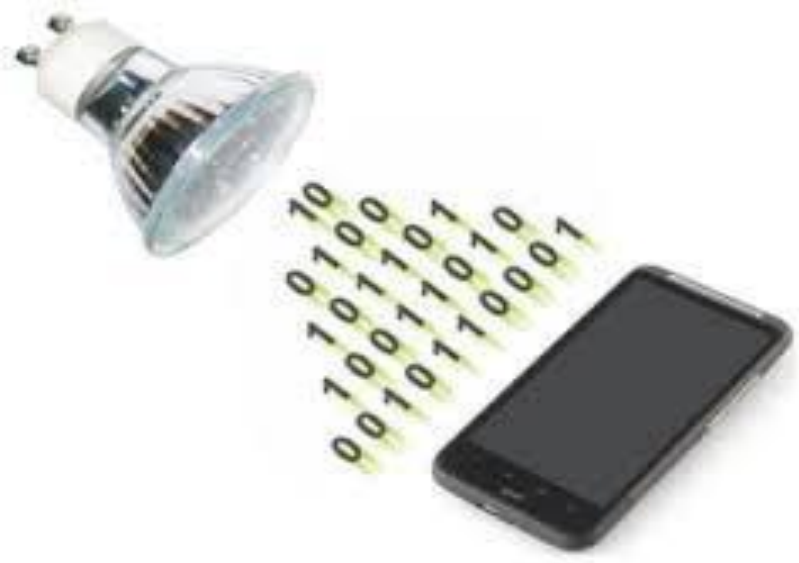

Fig 5: Overview of Li-Fi

\section{APPLICATIONS OF LI-FI}

\subsection{Underwater communication}

As the waves are intensely immersed by the sea water within the feet of their transmission. Hence, radio waves cannot use under water as well as it extracts it unusable underwater.

\subsection{Increase communication security}

Light cannot penetrate through wall, so Li-Fi technology is much more secure than any other communication system.

\subsection{Airways}

There is no internet access in the aircrafts. However aircrafts have numerous number of LED's present, thus data transmission is possible.

\subsection{Anywhere internet}

Accessing of the internet is possible anywhere where light source is available. 


\section{CHALLENGES FOR LI-FI}

In spite of having many advantages over Wi-Fi, Li-Fi technology is facing several delinquents such as the line of sight. Another disadvantage for Li-Fi is light cannot penetrate through walls as radio waves and is easily blocked by the person simply walking in front of LED light. A major challenge to the $\mathrm{Li}-\mathrm{Fi}$ is how to make Li-Fi system selfpowered as well as how the receiving scheme will transmit the data back to the transmitter.

\section{CONCLUSION}

This paper presents a review of the studies done on various aspects of Li-Fi. This may solve the issue of availability as well as security. Li-Fi is in initial stages, but enhancements are being made promptly, and soon this technology will be able to be used in our daily lives.

This ensures that the future of Li-Fi is bright as the real-time transmission is possible using solar cell.

\section{REFERENCES}

[1] Harald Haas, "Li-Fi is a paradigm-shifting 5G technology." Reviews in Physics 3 (2018): 26-31.

[2] Soni, Nidhi, Mayank Mohta, and Tanupriya Choudhury. "The looming visible light communication $\mathrm{Li}-\mathrm{Fi}$ : An edge over Wi-Fi." System Modeling \& Advancement in Research Trends (SMART), International Conference. IEEE, 2016.

[3] Nivrutti, Dhakane Vikas, and Ravi Ramchandra Nimbalkar. "Light-Fidelity: A Reconnaissance of Future Technology." International journal of advanced research in computer science and software engineering 3.11 (2013)

[4] Sharma, Rahul R., and Akshay Sanganal. "Li-Fi Technology: Transmission of data through light." International Journal of Computer Technology and Applications 5.1 (2014): 150.

[5] Prakash, Ravi, and Prachi Agarwal. "The New Era of Transmission and Communication Technology: Li-Fi (Light Fidelity) LED \& TED Based Approach." International Journal of Advanced Research in Computer Engineering \& Technology (IJARCET) 3.2 (2014).
[6] Bhut, Jay H., Dharmrajsinh N. Parmar, and Khushbu V. Mehta. "LI-FI Technology-A Visible Light Communication." (2014).

[7] Haruyama, Shinichiro. "Visible light communication using sustainable LED lights." ITU Kaleidoscope: Building Sustainable Communities (K-2013), 2013 Proceedings of. IEEE, 2013.

[8] McColl, Shelley L., and Jennifer A. Veitch. "Fullspectrum fluorescent lighting: a review of its effects on physiology and health." Psychological medicine 31.6 (2001): 949-964.

[9] Mutthamma, M. "A survey on Transmission of data through illumination-Li-Fi." IJRCCT 2.12 (2013): 1427 1430 .

[10] Lee, Jin-Shyan, Yu-Wei Su, and Chung-Chou Shen. "A comparative study of wireless protocols: Bluetooth, UWB, Zig-Bee, and Wi-Fi." Industrial Electronics Society, 2007. IECON 2007. 33rd Annual Conference of the IEEE, 2007.

[11] Mahendran, R. "Integrated Li-Fi (Light Fidelity) for smart communication through illumination." Advanced Communication Control and Computing Technologies (ICACCCT), 2016 International Conference on. IEEE, 2016.

[12] Afgani, Mostafa Z., et al. "Visible light communication using OFDM." Test beds and Research Infrastructures for the Development of Networks and Communities, 2006. TRIDENTCOM 2006. 2nd International Conference on. IEEE, 2006.

[13] Mohit vasuja,et.al. “ Image transmission using Li-Fi”. Proceedings of the 2nd International Conference on Inventive Communication and Computational Technologies (ICICCT 2018). IEEE Xplore Compliant Part Number: CFP18BAC-ART; ISBN:978-1-53861974-2

[14] Sonnad, Akshata M., et al. "Recent advancements in LiFi technology." International Journal of Electrical, Electronics, and Data Communication 1.10 (2013): 6163. 